\title{
SOME PROPERTIES OF THE DISCRIMINANT MATRICES OF A LINEAR ASSOCIATIVE ALGEBRA*
}

BY R. F. RINEHART

1. Introduction. Let $A$ be a linear associative algebra over an algebraic field. Let $e_{1}, e_{2}, \cdots, e_{n}$ be a basis for $A$ and let $c_{i j k}$, $(i, j, k=1,2, \cdots, n)$, be the constants of multiplication corresponding to this basis. The first and second discriminant matrices of $A$, relative to this basis, are defined by

$$
\begin{aligned}
& T_{1}(A)=\left\|t_{1}\left(e_{r} e_{s}\right)\right\|=\left\|\sum_{i, j=1}^{n} c_{r s i} c_{i j}\right\|=\left\|\sum_{i, j=1}^{n} c_{r i j} c_{s j i}\right\|, \\
& T_{2}(A)=\left\|t_{2}\left(e_{r} e_{s}\right)\right\|=\left\|\sum_{i, j=1}^{n} c_{r s i} c_{j i j}\right\|=\left\|\sum_{i, j=1}^{n} c_{i r j} c_{j s i}\right\|,
\end{aligned}
$$

where $t_{1}\left(e_{r} e_{s}\right)$ and $t_{2}\left(e_{r} e_{s}\right)$ are the first and second traces, respectively, of $e_{r} e_{s}$. The first forms in terms of the constants of multiplication arise from the isomorphism between the first and second matrices of the elements of $A$ and the elements themselves. The second forms result from direct calculation of the traces of $R\left(e_{r}\right) R\left(e_{s}\right)$ and $S\left(e_{r}\right) S\left(e_{s}\right), R\left(e_{i}\right)$ and $S\left(e_{i}\right)$ denoting, respectively, the first and second matrices of $e_{i}$. The last forms of the discriminant matrices show that each is symmetric.

E. Noether $\dagger$ and C. C. MacDuffee $\ddagger$ discovered some of the interesting properties of these matrices, and shed new light on the particular case of the discriminant matrix of an algebraic equation. It is the purpose of this paper to develop additional properties of these matrices, and to interpret them in some familiar instances.

Let $A$ be subjected to a transformation of basis, of matrix $M$,

$$
e_{i}=\sum_{j=1}^{n} m_{i j} e_{j}, \quad\left(i=1,2, \cdots, n ; m=\left|m_{r s}\right| \neq 0\right) .
$$

* Presented to the Society, November 30, 19.35. This paper, with proofs and details not included here, is on file as a doctoral thesis in the Library of the Ohio State University.

$\dagger$ Mathematische Zeitschrift, vol. 30 (1929), p. 689.

$\ddagger$ Annals of Mathematics, (2), vol. 32 (1931), pp. 60-66; and Transactions of this Society, vol. 33 (1931), pp. 425-432. 
MacDuffee noted* that under such a transformation the discriminant matrices are transformed by

$$
T_{1}=M T_{1}^{\prime} M^{T}, \quad T_{2}=M T_{2}^{\prime} M^{T}
$$

where $M^{T}$ denotes the transpose of $M$; that is, these matrices are transformed in the same way as are the matrices of quadratic forms. Thence he proved that, if $A$ has a principal unit, either discriminant matrix may be reduced to a diagonal form, $\left\|g_{r} \delta_{r s}\right\|$, by means of a transformation of basis which leaves the principal unit invariant. Further, for this normal basis the constants of multiplication satisfy the cyclic relations

$$
g_{p} c_{r i p}=g_{i} c_{p r i}, \quad(i, p, r=1,2, \cdots, n) .
$$

As a consequence of (1) he proved that for every choice of basis

$$
S(x) T_{i}=T_{i} R(x), \quad(i=1,2),
$$

for every element $x$ of $A$. In a subsequent paper L. E. Bush $\dagger$ extended MacDuffee's results to a general associative algebra.

2. Another Reduction to Normal Form. It is possible to derive the normal forms of the discriminant matrices of a general algebra by a method essentially simpler than that of Bush. Since no reduction is necessary if $A$ is nilpotent, let $A$ be non-nilpotent. Then $A$ is the sum of a semi-simple component $B$ and its radical $N$, which may be zero. Let $e_{1}, \cdots, e_{p}, e_{p+1}, \cdots, e_{n}$ be such a basis for $A$ that $e_{p+1}, \cdots, e_{n}$ constitute a basis for $N$ and $e_{1}, \cdots, e_{p}$ a basis for $B, e_{1}$ being the principal unit of $B$. Since both traces of a nilpotent element are zero, and since $N$ is invariant in $A$,

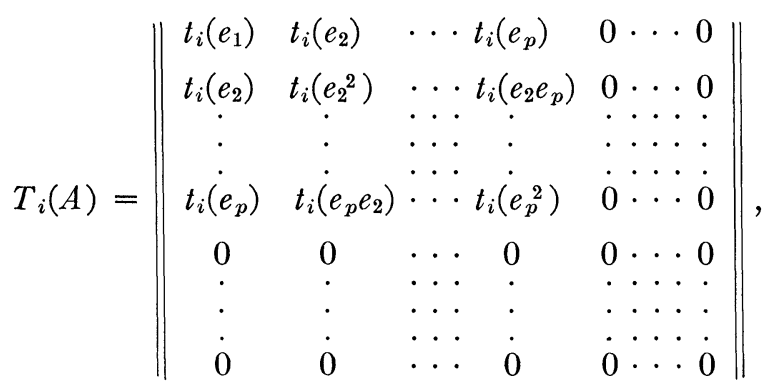

* This property was earlier discovered by E. Noether, loc. cit.

$\dagger$ This Bulletin, vol. 38 (1932), pp. 49-51. 
$(i=1,2)$. Since $e_{1}$ is idempotent and $F$ is non-modular, $t_{i}\left(e_{1}\right) \neq 0$, $(i=1,2)$. Hence by a transformation of basis of $A$ which leaves the basis of $N$ unchanged and the principal unit of $B$ invariant, $T_{1}(A)$ [or $\left.T_{2}(A)\right]$ can be reduced to a diagonal form $\left\|g_{r} \delta_{r s}\right\|$, where $g_{i}=0,(i>p)$. A manipulation of the associativity conditions for this normal basis yields (1) from which (2) may be derived.* From these relations we derive the following corollaries.

Corollary 1. For every semi-simple algebra over the complex field, a basis can be so chosen that the first and second matrices of each element of the algebra are equal.

Corollary 2. If $A$ is a commutative semi-simple algebra over the complex field, a basis for $A$ can be so chosen that the first and second matrices of each element of $A$ are equal and symmetric.

For over the complex field a normal basis may be so chosen that $T(A)$ is scalar, whence (2) yields $S(x)=R(x)$. If $A$ is also commutative, then $c_{i j k}=c_{j i k}$ and

$$
\begin{aligned}
R^{T}(x)=\subseteq \boldsymbol{T}(x) & =\sum_{i=1}^{n} x_{i}\left\|c_{r i s}\right\|^{T}=\sum_{i=1}^{n} x_{i}\left\|c_{s i r}\right\| \\
& =\sum_{i=1}^{n} x_{i}\left\|c_{i s r}\right\|=R(x)=S(x) .
\end{aligned}
$$

The existence of a normal basis can be applied to give a matric proof of the fact that a semi-simple algebra is the direct sum of simple algebras, and conversely. Let $A$ be a semi-simple algebra with a proper invariant subalgebra $B$. Then if $e_{1}, \cdots, e_{p}$, $e_{p+1}, \cdots, e_{n}$ is a normal basis for $A$, and $e_{1}, \cdots, e_{p}$ the corresponding basis for $B$, the first and second matrices of the basal elements are

$$
\begin{array}{lll}
R\left(e_{i}\right)=\left\|\begin{array}{cc}
R_{B}\left(e_{i}\right) & M_{i} \\
0 & 0
\end{array}\right\|, & S\left(e_{i}\right)=\left\|\begin{array}{cc}
S_{B}\left(e_{i}\right) & 0 \\
M_{i}^{\prime} & 0
\end{array}\right\|, & (i \leqq p), \\
R\left(e_{i}\right)=\left\|\begin{array}{cc}
P_{i} & Q_{i} \\
0 & L_{i}
\end{array}\right\|, & S\left(e_{i}\right)=\left\|\begin{array}{cc}
P_{i}^{\prime} & 0 \\
Q_{i}^{\prime} & L_{i}^{\prime}
\end{array}\right\|, & (i>p),
\end{array}
$$

where $R_{B}\left(e_{i}\right)$ and $S_{B}\left(e_{i}\right)$ denote, respectively, the first and sec-

* It should be noted that the restriction on the field $F$ is necessary only for the reduction to normal form; by direct verification it is easily seen that (2) holds for any basis, regardless of the nature of $F$. 
ond matrices of $e_{i}$, the representation being based on the algebra $B$ only. Further, for this basis,

$$
T_{1}(A)=\left\|\begin{array}{cc}
T_{1}(B) & 0 \\
0 & W
\end{array}\right\|
$$

where $T_{1}(B)$ and $W$ are non-singular. Hence, applying (2),

$$
M_{i}=M_{i}^{\prime}=0, \quad(i \leqq p) ; \quad Q_{i}=Q_{i}^{\prime}=0, \quad(i>p) .
$$

By means of the cyclic relations we find further that $P_{i}=P_{i}^{\prime}=0$, $(i>p)$. Hence

$$
R(x)=\left\|\begin{array}{cc}
R_{B}(x) & 0 \\
0 & R_{C}(x)
\end{array}\right\|, \quad S(x)=\left\|\begin{array}{cc}
S_{B}(x) & 0 \\
0 & S_{C}(x)
\end{array}\right\| .
$$

Obviously the system of matrices $R_{c}(x)\left[S_{c}(x)\right]$ is closed under addition and multiplication, and the $L_{i}\left[L_{i}^{\prime}\right]$ are linearly independent. Hence the linear system $\left(e_{p+1}, \cdots, e_{n}\right)$ is an algebra,* and therefore $A=B+C, \dagger$ where $B$ and $C$ are semi-simple algebras. This process can be continued with $B$ and $C$ in place of $A$, and so on, until $A$ is decomposed into simple components. That the decomposition is unique may be proved as in Dickson's Algebren und ihre Zahlentheorie.

The converse of this theorem is immediate from the standpoint of the discriminant matrix. For the first discriminant matrix of a direct sum of algebras is the direct sum of the first discriminant matrices of the component algebras, $\ddagger$ whence, if each component is simple, and non-null, each of the component matrices is non-singular, and therefore so is the direct sum.

3. Equality of $T_{1}$ and $T_{2}$. Since $T_{1}$ and $T_{2}$ are transformed cogrediently, their equality is an invariant property of $A$. If $A$ is nilpotent, then $T_{1}(A)=T_{2}(A)=0$. Suppose then that $A$ is non-nilpotent, with the basis $e_{1}, \cdots, e_{p}, \cdots, e_{n}$, where $e_{p+1}, \cdots, e_{n}$ constitute a basis for the radical (if any), and $e_{1}, \cdots, e_{p}$ a basis for the semi-simple component of $A$. For this

* Since $A$ is semi-simple, it is equivalent to the algebra of its first [second] matrices.

$\dagger+$ denotes direct sum.

$\ddagger$ MacDuffee, Annals of Mathematics, loc. cit. 
basis $T_{1}$ and $T_{2}$ are in the form (2). Hence an obvious necessary condition that $T_{1}=T_{2}$ is

$$
t_{1}\left(e_{r}\right)=t_{2}\left(e_{r}\right), \quad(r=1,2, \cdots, p) .
$$

This condition is clearly sufficient, since (4) implies

$$
t_{1}\left(e_{r} e_{s}\right)=\sum_{i=1}^{p} c_{r s i} t_{1}\left(e_{i}\right)=\sum_{i=1}^{p} c_{r s i} t_{2}\left(e_{i}\right)=t_{2}\left(e_{r} e_{s}\right) .
$$

Now (4) implies that $t_{1}(x)=t_{2}(x)$ for every $x$ of $A$, and conversely. Hence if $x$ is any element of $A$, and if $T_{1}=T_{2}$, then $t_{1}\left(x^{i}\right)=t_{2}\left(x^{i}\right)$ for every $i$. By a theorem of Frobenius* this implies that

$$
s_{i}=s_{i}^{\prime}, \quad(i=1,2, \cdots, n),
$$

$s_{i}$ and $s_{i}^{\prime}$ being the sum of the $i$ th powers of the first and second characteristic roots, respectively, of $x$. By Newton's identities this implies that the first and second characteristic functions of $x$ are equal. Conversely, if the characteristic functions of each element $x$ are equal, then so are the two traces of $x$.

THEOREM 1. The discriminant matrices are equal if, and only if, the characteristic functions of each element of the algebra are equal.

If $A$ is semi-simple, then $T_{1}(A)$ is non-singular and (2) may be written $S(x)=T_{1} R(x) T_{1}^{\mathrm{I}}$; hence, $S(x)$ and $R(x)$ being similar, the characteristic functions are equal. This proves the following corollary.

Corollary. If $A$ is semi-simple, $T_{1}(A)=T_{2}(A) . \dagger$

By a further elementary analysis, which is omitted here, it may be shown that: The equality of $T_{1}(A)$ and $T_{2}(A)$, when $A$ is neither semi-simple nor nilpotent, depends only on the multiplicative relations existing between the radical of $A$ and any semi-simple component $B$ of $A$. In particular, if $N$ is commutative with $B$, then $T_{1}(A)$ is equal to $T_{2}(A)$.

C. C. MacDuffee $\ddagger$ defined $\rho(\sigma)$ to be the maximum number of linearly independent linear relations among the first matrices $R\left(e_{i}\right)\left[S\left(e_{i}\right)\right]$ of an algebra. and proved that $\rho$ is equal to the

* G. Frobenius, Journal für Mathematik, vol. 51 (1856), pp. 209-271.

$\dagger$ Proved by MacDuffee by manipulation of the cyclic relations.

$\ddagger$ This Bulletin, vol. 35 (1929), pp. 344-349. 
order of the maximal zero subalgebra $Z$, such that $Z A=0$, and $\sigma$ is equal to the order of a similar algebra $W$, such that $A W=0$. By a long but elementary analysis omitted here, the following theorem can be proved.

THEOREM 2. If $A$ is a non-nilpotent algebra with a commutative radical $N$, a necessary and sufficient condition that $\rho=\sigma$ is that $t_{1}(u)=t_{2}(u)$, where $u$ is any principal idempotent of $A$.

Corollary. If $A$ is non-nilpotent and has a commutative radical, and if $T_{1}(A)=T_{2}(A)$, then $\rho=\sigma$.

It can be shown by examples that the condition $t_{1}(u)=t_{2}(u)$ is in general neither necessary nor sufficient, if $N$ is not commutative.

4. The Discriminant Matrix of an Algebraic Equation. We shall now consider two applications of the foregoing theory to the case of the commutative algebra generated by an algebraic equation. Let

$$
f(x)=x^{n}+a_{n-1} x^{n-1}+\cdots+a_{0}=0
$$

be an equation with coefficients in a field $F$. Let $A$ be the ring consisting of all polynomials with coefficients in $F$, reduced modulo $f(x)$, or, what is the same thing, the algebra generated by an element $x$, whose minimum equation is (5). For the customary basis $1, x, x^{2}, \cdots, x^{n-1}$, the discriminant matrix of $A$ is*

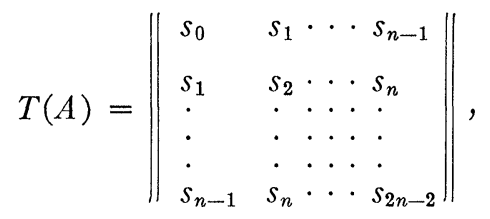

where $s_{i}$ is the sum of the $i$ th powers of the roots of (5), $s_{0}$ being defined to be $n$.

If we form the equality (2) and equate corresponding elements of the resulting matrices, discarding the immediate identities, we obtain

$$
-\sum_{i=0}^{n-1} a_{i} s_{i+j}=s_{n+j}, \quad(j=0,1, \cdots, n-2),
$$

* MacDuffee, Annals of Mathematics, loc. cit. 
which are Newton's identities for $s_{k},(k=n, n+1, \cdots, 2 n-2)$. Again, if we form $S\left(x^{2}\right) T=T R\left(x^{2}\right)$, we get the additional identity for $s_{2 n-1}$. Similarly, if $(2)$ is applied with $x^{3}, x^{4}, \cdots$, all the Newtonian identities for $s_{k},(k \geqq n)$ are obtained. Conversely, Newton's identities for $k \geqq n$ imply that $S(x) T=T R(x)$. Thus (2) may be considered as an extension of Newton's identities to a general algebra.

The second application has to do with the following theorem.

BorchARDT-JACOBI THEOREM. If $f(x)=0$ is an algebraic equation with real coeffcients, the number of its distinct roots is equal to the rank of the discriminant matrix, and the number of its distinct real roots is equal to the signature of this matrix.*

We shall outline a proof of the first half of this theorem, using the discriminant matrix from the standpoint of linear algebra. Let

$$
f(x)=\prod_{i=1}^{m}\left(x-\alpha_{i}\right)^{p_{i}}=0
$$

be an algebraic equation with the distinct roots $\alpha_{1}, \cdots, \alpha_{m}$. Let $m<n$ and let $A$ be the algebra defined by $f(x)=0 . \dagger$ If we define $P(x)=\prod_{i=1}^{m}\left(x-\alpha_{i}\right)$, the elements of the set of polynomials

$$
P(x), x P(x), \cdots, x^{n-m-1} P(x),
$$

are linearly independent and each one (hence any linear combination of them) is nilpotent. It is easily seen that any nilpotent polynomial must contain the factor $P(x)$, and consequently the set (7) is a maximal such set and therefore constitutes a basis for the radical of $A$. Hence the discriminant matrix of $A$ is of nullity $n-m$, that is, of rank $m$. If $m=n$, it can be shown that there are no nilpotent elements, and hence no radical in $A$, whence the rank of the discriminant matrix is $n$.

Ashland College, Ashland, OHio

* A. Loewy, Ostwald's Klassiker der exakten Wissenschaften, No. 143, pp. 50-63.

$\dagger$ The ground field $F$ is here taken to be the complex field. The same proof, with only slight modifications, can be carried through if $F$ is considered to be any subfield of the complex field. 